of stars of increasing, as well as stars of decreasing, temperature, as has so long been advocated by Sir Norman Lockyer. Chiefly through the work of Hertzsprung and Russell, it has, in fact, been found that many well-known stars are in a highly diffuse state, and when such stars contract under the influence of their own gravitation, they must rise in temperature until they cease to approximate to perfectly gaseous conditions (density 0.2 to 0.4 compared with water). Prof. Eddington has made an important modification of Lane's theory by taking account of radiation-pressure, and he shows that as a star contracts the diminishing surface is compensated by increasing radiation, so that the total radiation remains nearly constant, until the density becomes too great for the theory to apply. Afterwards the star passes to the descending branch of the temperature curve, and the total radiation falls off very rapidly. Radiation-pressure has further been found to provide a reasonable explanation of the approximate uniformity of stellar masses.

\section{THE VISCOSITY OF SLAG AT HIGH TEMPERATURES.}

A LTHOUGH the data which have in recent years $\mathrm{A}$ been accumulated on the behaviour of silicates at high temperatures possess a great interest and value from the points of view of the mineralogist and the geophysicist, a knowledge of the melting points and fields of stability of the silicates is not the most impor. tant factor for consideration in so far as application to the metallurgy of iron is concerned. In a study of the blast-furnace process what is of particular interest is the behaviour of the slag from the time it enters the zone of fusion until it is flushed from the slag-notch. In passing through this region where the smelting process occurs the most important physical property of the slag is its viscosity, while its most important chemical property is its desulphurising power.

It has long been known to furnacemen that molten blast-furnace slag is much more viscous than molten iron and most fused salts, and that the slag undergoes a gradual softening on heating rather than a sudden change to a mobile liquid. This particular characteristic was from the first rightly attributed to the silica content of the slag, and it appears to be due to the nature of the molecule, $\mathrm{SiO}_{2}$, rather than to the element silicon itself. X-ray analysis in the hands of Prof. W. H. and Mr. W. L. Bragg has furnished an important confirmation of this hypothesis. Instead of finding, as is the case with the crystals of most chemical compounds, that the atoms are arranged separately at definite points of a space-lattice, they concluded that three silica molecules are associated with each point of the space-lattice. It is a matter of common knowledge that highly associated or polymerised liquids possess unusually high viscosity, and hence it seems plausible to argue that, since silica appears to be unusually complex in the solid state, this association or polymerisation tendency must be the fundamental cause of the extreme viscosity of silica itself and the high viscosity of the silicates in the liquid state. When a silicate gradually softens with rising temperature and passes entirely over into the liquid state it is probable that the increased fluidity is due to a weakening of the "residual-valence" attraction between the group-molecules, while the relatively high viscosity of the melt, as compared with that of molten metals and ordinary salts, is due to the preponderance of the group-molecules silica, alumina, and lime, and possibly in a particularly large degree to a highly polymerised condition of the silica group-molecule.

NO. 25 I5, VOL. IOO]
Considerations of this kind are set out in a valuable paper by Mr. A. L. Feild, assistant metallurgist at the United States Bureau of Mines, in a recent paper presented to the Faraday Society. ${ }^{\mathrm{I}}$ Mr. Feild points out that while it is theoretically possible to render any silicate mixture whatever sufficiently fluid to flow from the slag-notch of a blast-furnace it is necessary in practice that the slag should attain this necessary fluidity at a temperature which is not beyond the working limit of the blast-furnace lining, and does not demand an unusually high fuel consumption. It is obvious that if, for instance, a slag requires a minimum temperature of $1400^{\circ} \mathrm{C}$. in order to attain a working fluidity, no iron will be produced in a furnace using this slag, regardless of the number of B.Th. units developed within the furnace, unless the temperature distribution is such that the slag acquires the necessary temperature at $1400^{\circ} \mathrm{C}$. Thus the fuel economy of the blast-furnace is to a great extent dependent upon the temperature-viscosity relations of the slag. Apart from the question of mining cost and freightage, the value of an iron ore sufficiently rich in iron to be considered marketable largely depends on whether it can be made to yield economically a slag of desirable viscosity and desulphurising power.

Mr. Feild has worked out a method of determining the viscosities of slags up to $1600^{\circ} \mathrm{C}$., this limit being imposed by the furnace refractories and not by inherent limitations of the apparatus. He has used a modification of the method originated by Margules in $\mathrm{I} 88 \mathrm{I}$, in which the liquid is confined between two concentric cylinders. The outer cylinder is rotated at a constant speed, and the torque exerted upon the inner cylinder measured. The method is applicable to liquids of a wide range of viscosity, and has been applied in this case to measurements on slags over a range of viscosity from 200 to 3000 (water at $20^{\circ} \quad C=I$ ). Acheson graphite was used in the construction of all parts subjected to high temperatures, and the suspended system was damped so as to oive it the stability and aperiodicity of the familiar damped D'Arsonval galvanometer. The outer cylinder was rotated about a vertical axis at a constant speed. The inner cylinder was suspended coaxially within the outer one by means of a steel or phosphor-bronze ribbon. Experimental data derived by this method are capable of an easy direct mathematical interpretation. A cylinder of radius $b$, rotating with a constant angular velocity $\omega$, will exert upon an inner fixed concentric cylinder of radius $a$-the space between them being filled with the liquid-a couple $T$ given by the relation

$$
\Gamma=4 \pi \eta L^{2} \frac{a^{2} b^{2}}{b^{2}-a^{2}}
$$

where $\eta$ is the coefficient of viscosity and L the common length of the two cylinders. With cylinders of fixed dimensions the viscosity is proportional to the torsion couple and inversely proportional to the speed of rotation in revolutions per second. Conversely the torsion couple is proportional to the speed of rotation and to the viscosity.

Viscosity values are given for eight commercial slags, two synthetic slags, and an artificial diopside. The temperature-viscosity curve approximates in form to that of the rectangular hyperbola, while the temperature-fluidity curve approaches a straight line in form. The average viscosity at $1500^{\circ} \mathrm{C}$. of eight commercial slags was found to be $30 \mathrm{I}$ (water at $20^{\circ} \mathrm{C}=1$ ). Refractory slags have been found to be not necessarily more viscous at high temperature than more fusible ones.

H. C. H. C.

1 "The Viscosity of Blast-furnace Slag and its Relation to Iron Metallurgy, including a Description of a New Method of Measuring Slag Viscosity
at High Temperatures." Proceedings, December, 1917. 